\title{
Energy-aware Adaptive Multimedia for Game-based E-learning
}

\author{
Ioana Ghergulescu, Arghir-Nicolae Moldovan and Cristina Hava Muntean \\ School of Computing, National College of Ireland \\ Mayor Street, IFSC, Dublin 1, Ireland \\ e-mail: ioana.ghergulescu@ncirl.ie; amoldovan@student.ncirl.ie; cristina.muntean@ncirl.ie
}

\begin{abstract}
Thanks to their motivational potential, video games have started to be increasingly used for e-learning. However, as e-learning gradually shifts towards mobile learning, there is a growing need for innovative techniques to deliver rich learning material such as educational games to resource-constrained devices. In particular, the limited battery capacity of mobile devices stands out as a key issue that can significantly limit players' access to educational games. This paper proposes an Energy-aware Adaptive Multimedia Game-based E-learning (EAMGBL) framework that aims to enable energy-efficient educational games delivery to mobile devices over wireless networks. The framework builds on top of the idea to render the game on the server side and stream a recording of it to the player's device over the Internet. To reduce the mobile device energy consumption and enable the player to play for longer, the proposed framework proposes to adapt both the educational game elements as well as the game's recorded multimedia stream.
\end{abstract}

Keywords-multimedia content adaptation, educational video games, energy consumption, quality of experience (QoE)

\section{INTRODUCTION}

E-learning, or the use of digital technologies to facilitate learning, has seen much evolution over the past few years, as a result of technological advances as well as having to keep up with the needs of $21^{\text {st }}$ century learners in a fast changing world. In particular, e-learning has started to integrate video games in the learning process due to their potential to support higherorder learning outcomes, to engage the new generation of digital learners and to bridge the gap between their expectations and traditional learning practices [1]-[3].

At the same time, users in general and learners in particular are increasingly adopting a mobile Internet connected lifestyle, with yearly global smartphone sales in particular having already crossed the 1 billion milestone [4]. However, the high variability of mobile device characteristics and capabilities poses new challenges to online service providers.

Recently, there has been a fast growth of game streaming services that deliver on the promise of cloud computing to enable playing of complex 3D games from a wide variety of devices with different characteristics and capabilities, even from resource-constrained ones. Rendering the game on the server and streaming a recording of it to the player's mobile device over the Internet takes significant workload out of the

This work was funded in part by the Irish Research Council EMBARK Postgraduate Scholarship Scheme. device. However, new issues and challenges have to be addressed in terms of various aspects such as user responsiveness, audio/video quality, service quality, operating cost or game adaptation [5], [6].

The limited battery capacity in particular, stands out to be the major key limitation of mobile devices [7]-[11], as it can significantly limit players' access to educational material and affect their experience with the game-based e-learning system, especially when they have to interrupt their game-play due to low battery situations. Moreover, game streaming puts significant strain on the device battery, which has to power at the same time multiple power hungry components such as the wireless card, CPU and display in order to receive, decode and display the multimedia stream.

Therefore, a key challenge is the adaptation of the streamed games to enable energy saving while at the same time a good user Quality of Experience (QoE) [12]-[14]. The adaptation can address the game elements, the multimedia stream or a combination of the two, and along with the available battery energy has to consider various other resource constraints such as for example, the download limit for optimum stream delivery [6].

In this context, this paper aims to propose and evaluate an Energy-aware Adaptive Multimedia Game-based E-learning (EAMGBL) framework that would enable energy-efficient streaming of video games in game-based e-learning systems.

The rest of the paper is structured as follows. Section II presents some research works related to this paper. Section III presents the proposed EAMGBL framework. Section IV presents the experimental testing setup and results. Section V concludes the paper and presents future work directions.

\section{RELATED WORK}

While extensive research work was conducted on mobile devices energy saving in general, significant less research was conducted on energy saving in mobile games in particular. Furthermore, to our best knowledge, no study had addressed energy saving in educational mobile games.

A number of different research papers have surveyed the various existing energy saving aspects and the existing energy saving solutions in mobile devices [15]-[19]. Moldovan et al. [15] have surveyed various approaches for energy measurement, modelling and adaptation, the three major 


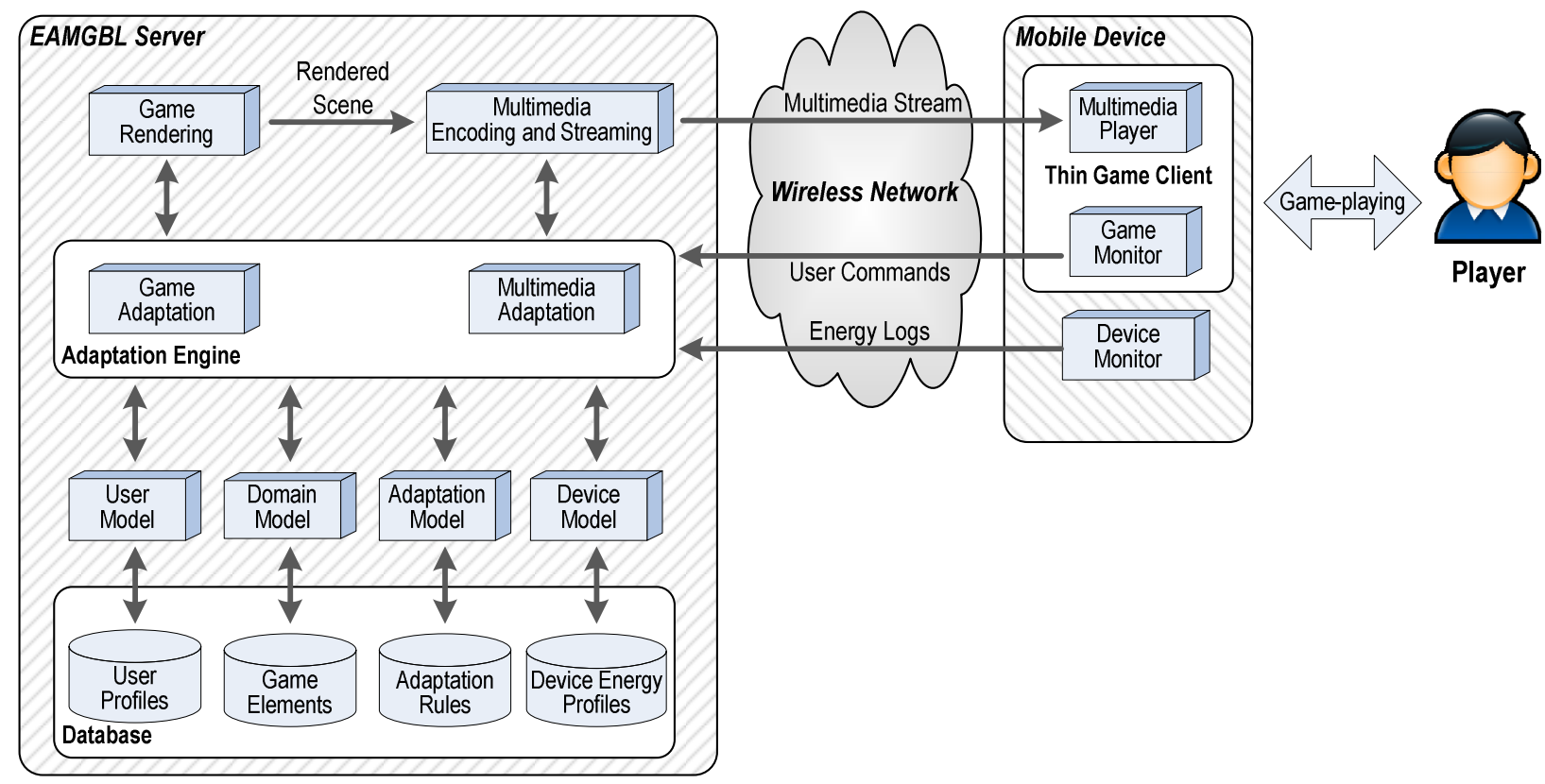

Fig. 1. Block level representation of the proposed Energy-aware Adaptive Multimedia Game-based E-learning (EAMGBL) framework.

aspects that have to be considered in order to deploy energyaware mobile learning systems and applications. VallinaRodriguez and Crowcroft [16] have addressed energy management techniques in mobile devices with focus on energy efficient operating systems (OS). The authors presented a number of energy-aware OSs, resource profilers and resource management techniques as well as solutions for optimizing energy consumption of wireless interfaces.

Energy-aware multimedia delivery was also the focus of several different surveys [17]-[19]. Starting from experimental tests conducted on a smartphone device, Kennedy et al. [17], have identified the major energy consuming components in high-end devices namely display, CPU and network interface, and continued by presenting and classifying existing multimedia-centric energy saving solutions for these components. Hoque et al. [18] have surveyed various solutions for improving the energy efficiency of wireless multimedia streaming in mobile devices. Zhang et al. [19] have surveyed existing energy saving techniques for mobile multimedia delivery, which were grouped by the authors in two main approaches: power aware video coding and video delivery.

One major direction for energy saving in mobile games streaming is putting the wireless interface in a low energy consumption state when it is not used. Harvey et al. [20] have proposed to reduce energy consumption in multi-player mobile games, by putting the wireless interface in a low energy consumption state based on dead reckoning errors, more specifically the difference between the predicted location of the game objects and their true location. The authors have tested their solution using a multiplayer game simulator, the results showing that their proposed solution could achieve up to $36 \%$ energy saving. The power consumption of the wireless interface in a multi-player game was also shown that it can be reduced with between $20 \%$ and $50 \%$ by employing an algorithm based on dynamic lookahead for putting the wireless interface in a sleep state [21].

Several energy saving solutions based on adapting the game elements or the game multimedia stream have also been proposed [6]. Game objects adaptation approaches include adapting their elements such as textures and lighting effects that are more bandwidth and rendering computation expensive, the by taking into consideration the object importance and bandwidth restrictions [22],[23]. On the same idea multimedia stream adaptation can be done for example by encoding different areas of a frame corresponding to different game elements at different quality [24], [25].

\section{EAMGBL FRAMEWORK}

Fig. 1 presents the block-level representation of the proposed Energy-aware Adaptive Multimedia Game-based Elearning (EAMGBL) framework, with the interaction between its main components. EAMGBL is based on the generic clientserver architecture of an Adaptive E-learning System (AELS) that consists of an Adaptation Engine and various models. The functionality and the components of the EAMGBL framework are briefly presented next.

The EAMGBL server comprises the following components: User Model, Domain Model, Adaptation Model, Device Model, Adaptation Engine, Game Rendering Module and Multimedia Recording-Encoding-Streaming Module.

The User Model stores information about the players in the form of user profiles. The information may include: demographic data (e.g., name, age, gender, etc.), playing expertise, game playing preferences, knowledge level on the studied material, motivation, etc. The user profiles are built automatically based on information received explicit from the user (e.g., through forms, questionnaires), or implicit through game-play monitoring. 
The Domain Model stores the individual game elements and the relationships between them, which are combined in order to create the in-game environment.

The Device Model maintains device energy profiles corresponding to the different mobile devices used by the players for accessing the educational games. The device profiles contain information about the mobile devices' characteristics and capabilities, such as: device model, operating system, screen size and resolution, CPU and GPU speed, memory, battery type and model, battery capacity, etc. Additionally, they are continuously updated with real-time information about the available battery energy that is collected through a Device Monitor located on the client-side.

The Game Rendering Module performs the computation intensive rendering tasks, enabling the players to use mobile devices with lower capabilities in order to play the educational games. The rendered game frames are recorded, compressed and streamed to the mobile device for playback.

The Adaptation Engine (AE) performs the energy-aware adaptation of the educational game. For maximising the energy saving, EAMGBL combines two types of adaptation: game elements adaptation and multimedia adaptation. The game elements are classified based on their importance for each game-playing scene, and the frames are divided into areas-ofinterest (AOIs). To reduce the amount of transmitted data and the device energy consumption, the AOIs corresponding to more important game elements are encoded at a higher quality than those corresponding to less important game elements.

On the client side, the player interacts with the game through a thin client. The Game Monitor is responsible with capturing the player's inputs, and passing them to the server where they are used in the actual control of the game.

\section{EXPERIMENTAL TESTING AND RESULTS}

\section{A. Educational Game}

The "Fire Protocol" [26] educational game developed using the e-Adventure game platform [27], was selected for testing purposes (see Fig. 2). Fire Protocol is a first-person game that aims to teach the fire safety evacuation protocol from a practical perspective. In the game, the player takes the role of Pablo, a member of the faculty. The game starts in the office where the players has to answer the phone, being informed to check for fire as the alarm was triggered in office 411 , and to start the evacuation procedure if necessary.

After checking for fire, the player has to activate the alarm and then to check the teacher offices. In one office the player
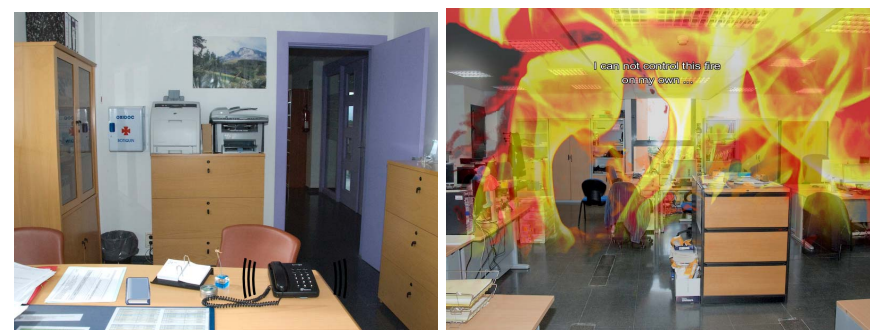

Fig. 2. Screenshots from the 'Fire Protocol' educational game.

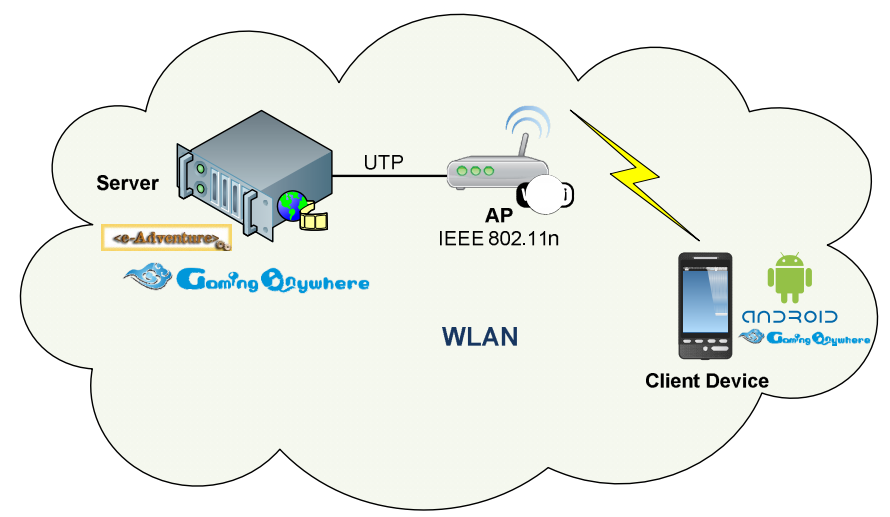

Fig. 3. Wireless test-bed setup.

has a conversation with another character, informing him that there is a fire in office 411 and he has to leave the building immediately. Once the player has checked all offices he can proceed to leave the building. In order to successfully complete the evacuation procedure the player has to do all the tasks, as well as to maintain his calm during the conversation and to leave the building on stairs rather than taking the elevator. The Fire Protocol game was extended to incorporate a methodology for player motivation monitoring in game-based e-learning through making use of motivation metrics and implemented using game events [28]-[30].

\section{B. Wireless Test-bed Setup}

Fig. 3 illustrates the experimental test-bed setup, consisting of a server, a wireless access point and a client device. The server machine was a Dell Vostro 3350 laptop PC running Microsoft Windows 7 64-bit operating system, and having a $2.3 \mathrm{GHz}$ Intel Core i5-2410M CPU and $8 \mathrm{~GB}$ of RAM. The client device was a Samsung Galaxy SII smartphone running on Google Android version 4.1.2 operating system, and having a 4.3" AMOLED capacitive touchscreen with a resolution of $800 \times 480$ pixels, an Exynos $42101.2 \mathrm{GHz}$ dual-core ARM Cortex-A9 CPU and 1 GB of RAM. The mobile device was capable to play full-HD 1080p videos without any buffering or playback issues.

In order to stream the "Fire Protocol" educational game, we used the open source cloud gaming system GamingAnywhere version 0.7.5 [31], [32]. The GamingAnywhere consists of a server application and a client application that are available for Windows, Linux and Mac OS X. Moreover, the client application was also ported to Android [33]. The game frames recorded on the server side are streamed to the GamingAnywhere client application using the RTSP over UDP protocol.

\section{Energy Measurement Setup}

External measurement equipment connected to the mobile device battery with the low-side current-shunt method [15], was used in order to collect accurate energy consumption measurements of the smartphone device during the game streaming. Fig. 4 illustrates the energy consumption measurement setup. An Arduino Uno board was used in order to read the voltage across the shunt resistor, as well as the 


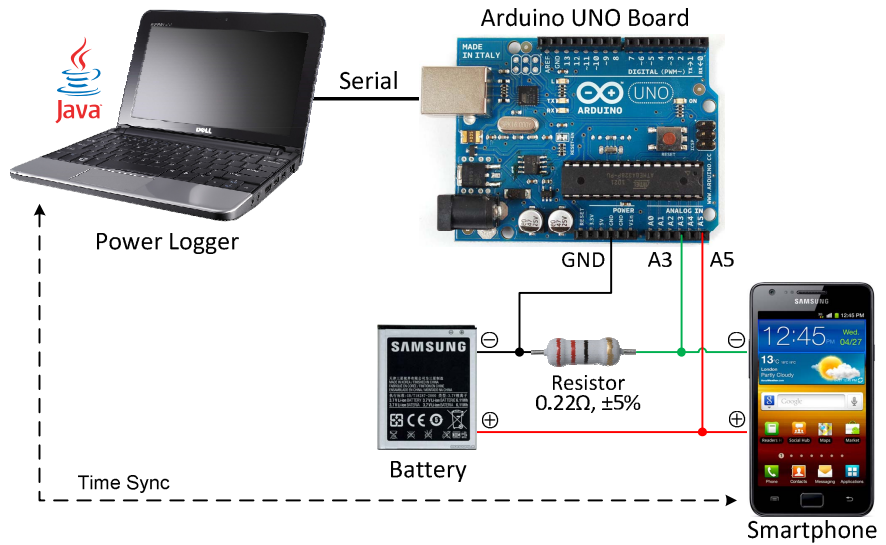

Fig. 4. Energy consumption measurement setup.

battery voltage. The voltage values represented with a 10-bit resolution are written to the serial port at a frequency of approximately $200 \mathrm{~Hz}$.

A Java application running on a Windows 7 laptop PC reads the voltage values from the serial port, and computes the average current load as well as the device power consumption for each second interval. The values are then saved to .csv files for further processing. The power logger PC and the smartphone device were time synchronized in order to facilitate the data analysis.

\section{Test Scenarios}

Table I presents the main encoding settings for the three testing scenarios that were considered in the experimental testing. The scenarios correspond to three different factors (i.e., video resolution, framerate and bitrate) that can be adapted in order to reduce the mobile device power consumption with retrieving, decoding and displaying the game recording multimedia streams.

The Fire Protocol educational game had a native resolution of $800 \times 600$ pixels. Five resolution values (i.e., $800 \times 600$, $640 \times 480,480 \times 360,320 \times 240$ and $240 \times 180$ pixels) were used for scenario 1 . The framerate was maintained to $30 \mathrm{fps}$, while the video bitrate was decreased with the resolution following the recommendations from [34].

Five video framerate values (i.e., $30,15,8,4$ and $2 \mathrm{fps}$ ) were considered for testing scenario 2. The resolution was maintained constant to $800 \times 600$ pixels for each test case. As opposed, the video bitrate was decreased with the decrease in framerate, while maintaining a constant quantization factor equal to 25 .

Five video bitrate values (i.e., 1500, 1200, 900, 600, 300 kbps) were considered for testing scenario 3 . The resolution was maintained constant to $800 \times 600$ pixels for each test case. The framerate was also maintained constant to $30 \mathrm{fps}$.

The GamingAnywhere system makes use of libavcodec from FFmpeg in order to record the game frames on the server side. The encoding is done using the MPEG-4 AVC / H.264 video codec and the mp3 audio codec. All the other video and audio encoding parameters were maintained constant for all test cases. The audio bitrate was set to $128 \mathrm{kbps}$, while the
TABLE I. Testing Scenarios EnCoding SETtings

\begin{tabular}{cccc}
\hline \hline $\begin{array}{c}\text { Testing } \\
\text { Scenario }\end{array}$ & $\begin{array}{c}\text { Resolution } \\
{[\text { pixels] }}\end{array}$ & $\begin{array}{c}\text { Framerate } \\
{[\mathbf{f p s}]}\end{array}$ & $\begin{array}{c}\text { Video Bitrate } \\
{[\mathbf{k b p s}]}\end{array}$ \\
\hline Scenario1 & $800 \times 600$ & 30 & 1500 \\
(Resolution Change) & $640 \times 480$ & & 1000 \\
& $480 \times 360$ & & 550 \\
& $320 \times 240$ & & 300 \\
& $240 \times 180$ & & 150 \\
\hline Scenario2 & $800 \times 600$ & $30,15,8,4,2$ & $\mathrm{QP}=25$ \\
(Framerate Change) & & & \\
\hline Scenario 1 & $800 \times 600$ & 30 & 1500,1200, \\
(Bitrate Change) & & & $900,600,300$ \\
\hline \hline
\end{tabular}

audio sampling frequency was set to $48 \mathrm{KHz}$. The GOP size was maintained to the default value of 48 , recommended by the creators of the GamingAnywhere system [32].

The FireProtocol game does not allow to change the video resolution, while the GamingAnywhere system does not include video resolution scaling functionalities. Therefore, the resolution scaling was done by scaling the rendered video frames before being recorded for streaming.

\section{E. Results}

The results of the experimental testing are presented in Figures 5, 6 and 7, for the video resolution, framerate and bitrate change scenarios respectively. The figures present the average power consumption of the mobile device for each test case. Additionally, the figures present the power saving achieved by decreasing the resolution, framerate or bitrate, computed as the percentage relative to the highest quality for each scenario.

The results for scenario 1 presented in Fig. 5 show that the smartphone's energy consumption decreases with the video resolution decrease. The highest power saving of $18.13 \%$ for this scenario is achieved when changing the video resolution from $800 \times 600$ pixels to $240 \times 180$ pixels.

The results for scenario 2 presented in Fig. 6 show that considerably more battery power could be saved by decreasing the video framerates. For example, reducing the framerate from $30 \mathrm{fps}$ to $2 \mathrm{fps}$, reduced the smartphone's power consumption by $44.11 \%$.

The results for scenario 3 presented in Fig. 7 show that the smartphone's energy consumption also decreases with the video bitrate decrease, although not as much as for the case of framerate and resolution decrease. The highest power saving of $12.46 \%$ for this scenario is achieved when changing the video bitrate from $1500 \mathrm{kbps}$ to $300 \mathrm{kbps}$.

\section{CONCLUSIONS AND FUTURE WORK}

This paper has proposed the Energy-aware Adaptive Multimedia Game-based E-learning (EAMGBL) framework that aims to enable energy-efficient delivery of educational games rendered on the server and streamed to mobile devices over wireless networks. To save the mobile device battery power, the framework adapts the quality of the video game stream. Experimental testing was conducted in order to assess 


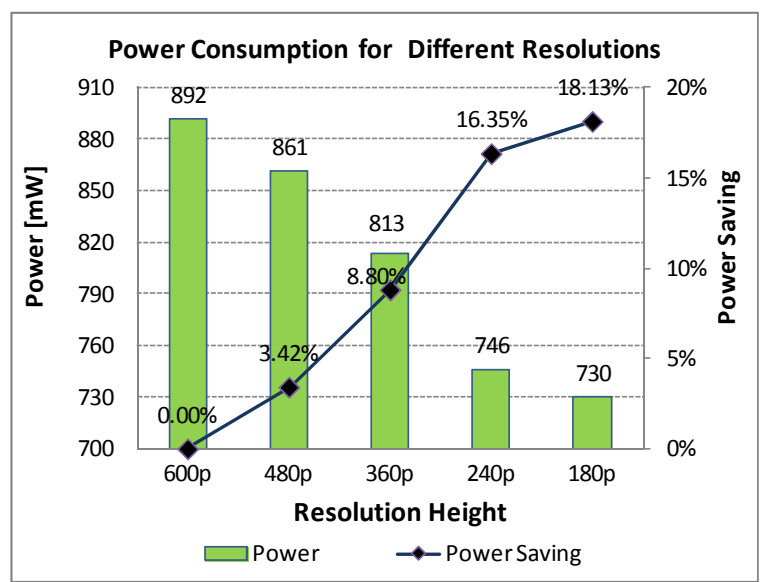

Fig. 5. Average power consumtion for different resolution values.

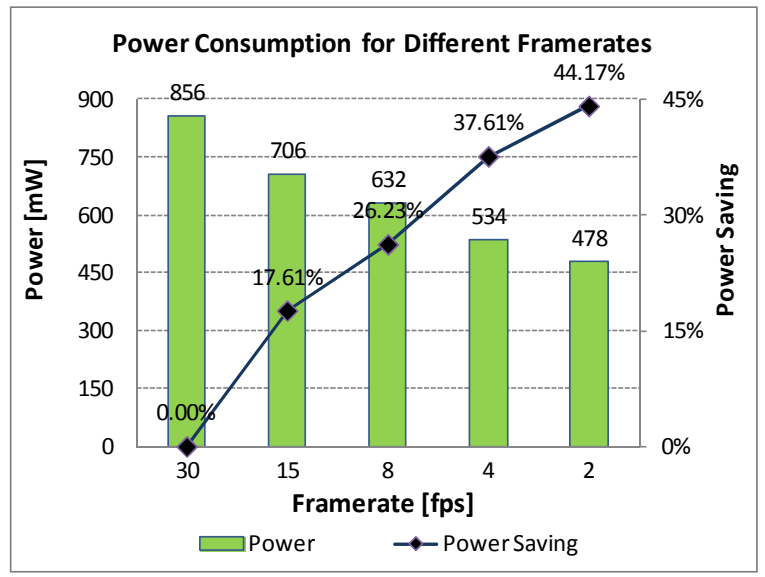

Fig. 6. Average power consumtion for different framerate values.

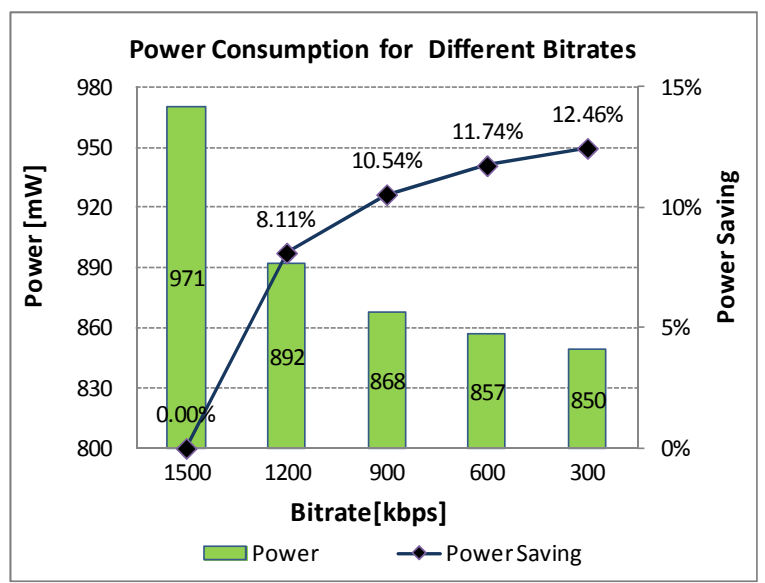

Fig. 7. Average power consumtion for different video bitrate values.

how much battery power can be saved by changing the encoding parameters such as video resolution, video bitrate and video framerate. The results have shown that decreasing the video framerate leads to the highest energy saving among the three parameters considered. Future work will extend the model with new techniques for adapting the game and the game-elements, such as region of interest-based adaptation.
Additional experimental and subjective testing will also be conducted.

\section{REFERENCES}

[1] M. Papastergiou, 'Exploring the potential of computer and video games for health and physical education: A literature review', Computers \& Education, vol. 53, no. 3, pp. 603-622, Nov. 2009.

[2] I. Ghergulescu and C. H. Muntean, 'Measurement and Analysis of Learner's Motivation in Game-Based E-Learning', in Assessment in Game-Based Learning, D. Ifenthaler, D. Eseryel, and X. Ge, Eds. New York, NY: Springer New York, 2012, pp. 355-378.

[3] I. Ghergulescu and C. H. Muntean, 'A Novel Sensor-Based Methodology for Learner's Motivation Analysis in Game-Based Learning', Interacting with Computers, p. iwu013, Apr. 2014.

[4] IDC, 'Worldwide Mobile Phone Market Forecast to Grow 7.3\% in 2013 Driven by 1 Billion Smartphone Shipments, According to IDC prUS24302813', 04-Sep-2013. [Online]. Available: http://www.idc.com/getdoc.jsp?containerId=prUS24302813. [Accessed: 04-Nov-2013].

[5] O. Soliman, A. Rezgui, H. Soliman, and N. Manea, 'Mobile Cloud Gaming: Issues and Challenges', in Mobile Web Information Systems, F. Daniel, G. A. Papadopoulos, and P. Thiran, Eds. Springer Berlin Heidelberg, 2013, pp. 121-128.

[6] S. Shirmohammadi, 'Adaptive Streaming in Mobile Cloud Gaming', IEEE COMSOC MMTC E-Letter, vol. 8, no. 5, pp. 20-22, 2013.

[7] A.-N. Moldovan, A. Molnar, and C. H. Muntean, 'EcoLearn: Battery Power Friendly e-Learning Environment for Mobile Device Users', in Learning-Oriented Technologies, Devices and Networks - Innovative Case Studies, A. Lazakidou and I. El Emary, Eds. Saarbrücken, Germany: LAP LAMBERT Academic Publishing, 2011, pp. 273-296.

[8] R. Trestian, A.-N. Moldovan, O. Ormond, and G.-M. Muntean, 'Energy Consumption Analysis of Video Streaming to Android Mobile Devices', in 13th IEEE/IFIP Network Operations and Management Symposium (NOMS 2012), Maui, Hawaii, USA, 2012, pp. 444-452.

[9] S. A. Jalal, N. Gibbins, D. Millard, and B. Al-Hashimi, 'Energy-aware adaptation of educational multimedia in mobile learning', presented at the 11th International Conference on Advances in Mobile Computing \& Multimedia (MoMM2013), Vienna, Austria, 2013.

[10] S. A. Jalal, N. Gibbins, D. Millard, B. Al-Hashimi, and N. R. Aljohani, 'Content-Aware Power Saving Multimedia Adaptation for Mobile Learning', in 2013 Seventh International Conference on Next Generation Mobile Apps, Services and Technologies (NGMAST), 2013, pp. 256-261.

[11] M. Kennedy, H. Venkataraman, and G.-M. Muntean, 'Battery and Stream-Aware Adaptive Multimedia Delivery for Wireless Devices', in 2010 IEEE 35th Conference on Local Computer Networks (LCN), IEEE International Workshop on Performance and Management of Wireless and Mobile Networks (P2MNET), Denver, Colorado, USA, 2010, pp. 843-846.

[12] A.-N. Moldovan and C. H. Muntean, 'Subjective Assessment of BitDetect - A Mechanism for Energy-Aware Multimedia Content Adaptation', IEEE Transactions on Broadcasting, vol. 58, no. 3, pp. 480-492, 2012.

[13] A.-N. Moldovan, I. Ghergulescu, S. Weibelzahl, and C. H. Muntean, 'User-centered EEG-based Multimedia Quality Assessment', in 8th IEEE International Symposium on Broadband Multimedia Systems and Broadcasting (BMSB 2013), Brunel University, Uxbridge, London, UK, 2013.

[14] A.-N. Moldovan and C. H. Muntean, 'Towards Personalised and Adaptive Multimedia in M-learning Systems', in Proceedings of 16th World Conference on E-Learning in Corporate, Government, Healthcare, and Higher Education (E-Learn 2011), Honolulu, Hawaii, USA, 2011, pp. 782-791.

[15] A.-N. Moldovan, S. Weibelzahl, and C. H. Muntean, 'Energy-Aware Mobile Learning: Opportunities and Challenges', IEEE Communications Surveys \& Tutorials, vol. 16, no. 1, pp. 234-265, 2014. 
[16] N. Vallina-Rodriguez and J. Crowcroft, 'Energy Management Techniques in Modern Mobile Handsets', IEEE Communications Surveys \& Tutorials, vol. 15, no. 1, pp. 179-198, 2013.

[17] M. Kennedy, A. Ksentini, Y. Hadjadj-Aoul, and G. Muntean, 'Adaptive Energy Optimization in Multimedia-Centric Wireless Devices: A Survey', IEEE Communications Surveys \& Tutorials, vol. 15, no. 2, pp. 768-786, 2013.

[18] M. A. Hoque, M. Siekkinen, and J. K. Nurminen, 'Energy Efficient Multimedia Streaming to Mobile Devices \#x2014; A Survey', IEEE Communications Surveys Tutorials, vol. 16, no. 1, pp. 579-597, 2014.

[19] J. Zhang, D. Wu, S. Ci, H. Wang, and A. K. Katsaggelos, 'Power-Aware Mobile Multimedia: a Survey (Invited Paper)', Journal of Communications, vol. 4, no. 9, pp. 600-613, Oct. 2009.

[20] R. C. Harvey, A. Hamza, C. Ly, and M. Hefeeda, 'Energy-efficient gaming on mobile devices using dead reckoning-based power management', in Network and Systems Support for Games (NetGames), 2010 9th Annual Workshop on, 2010, pp. 1-6.

[21] K. Thirugnanam, B. Anand, J. Sebastian, P. G. Kannan, A. L. Ananda, R. K. Balan, and M. C. Chan, 'Dynamic lookahead mechanism for conserving power in multi-player mobile games', in 2012 Proceedings IEEE INFOCOM, 2012, pp. 2721-2725.

[22] M. Hosseini, J. Peters, and S. Shirmohammadi, 'Energy-budgetcompliant Adaptive 3D Texture Streaming in Mobile Games', in Proceedings of the 4th ACM Multimedia Systems Conference, New York, NY, USA, 2013, pp. 1-11.

[23] M. Hosseini, A. Fedorova, J. Peters, and S. Shirmohammadi, 'Energyaware Adaptations in Mobile 3D Graphics', in Proceedings of the 20th ACM International Conference on Multimedia, New York, NY, USA, 2012, pp. 1017-1020.

[24] H. Ahmadi, S. Khoshnood, M. R. Hashemi, and S. Shirmohammadi, 'Efficient bitrate reduction using a Game Attention Model in cloud gaming', in 2013 IEEE International Symposium on Haptic Audio Visual Environments and Games (HAVE), 2013, pp. 103-108.

[25] M. Hemmati, A. Javadtalab, A. A. Nazari Shirehjini, S. Shirmohammadi, and T. Arici, 'Game As Video: Bit Rate Reduction Through Adaptive Object Encoding', in Proceeding of the 23rd ACM
Workshop on Network and Operating Systems Support for Digital Audio and Video, New York, NY, USA, 2013, pp. 7-12.

[26] P. Moreno-Ger, 'Fire Protocol', 2012. [Online]. Available: http://eadventure.e-ucm.es/course/view.php?id=29. [Accessed: 04-Jul-2013].

[27] J. Torrente, A. del Blanco, E. J. Marchiori, P. Moreno-Ger, and B. Fernandez-Manjon, '<e-Adventure $>$ : Introducing educational games in the learning process', in 2010 IEEE Education Engineering (EDUCON), 2010, pp. 1121-1126.

[28] I. Ghergulescu and C. H. Muntean, 'Learner Motivation Assessment with <e-Adventure> Game Platform', in World Conference on ELearning in Corporate, Government, Healthcare, and Higher Education 2011, 2011, vol. 2011, pp. 1212-1221.

[29] I. Ghergulescu and C. H. Muntean, 'Supporting Motivation Based Educational Games Through Web 3.0', in Towards Learning and Instruction in Web 3.0, P. Isaias, D. Ifenthaler, D. G. Sampson, and J. M. Spector, Eds. Springer New York, 2012, pp. 247-264.

[30] I. Ghergulescu, A.-N. Moldovan, and C. H. Muntean, 'Measurement of Self-Efficacy in Game-Based E-Learning Through Interaction With Non-Player Characters', in Proceedings of 25th Society for Information Technology and Teacher Education International Conference (SITE 2014), Jacksonville, Florida, USA, 2014, pp. 640-644.

[31] C.-Y. Huang, C.-H. Hsu, Y.-C. Chang, and K.-T. Chen, 'GamingAnywhere: An Open Cloud Gaming System', in Proceedings of the 4th ACM Multimedia Systems Conference, New York, NY, USA, 2013, pp. 36-47.

[32] C.-Y. Huang, K.-T. Chen, D.-Y. Chen, H.-J. Hsu, and C.-H. Hsu, 'GamingAnywhere: The First Open Source Cloud Gaming System', ACM Trans. Multimedia Comput. Commun. Appl., vol. 10, no. 1s, pp. 10:1-10:25, Jan. 2014.

[33] C.-Y. Huang, C.-H. Hsu, D.-Y. Chen, and K.-T. Chen, 'Quantifying User Satisfaction in Mobile Cloud Games', in Proceedings of Workshop on Mobile Video Delivery, New York, NY, USA, 2013, pp. 4:1-4:6.

[34] A.-N. Moldovan, I. Ghergulescu, and C. H. Muntean, 'Educational Multimedia Profiling Recommendations for Device-Aware Adaptive Mobile Learning', in 8th IADIS International Conference on e-Learning 2014 (eL2014), Lisbon, Portugal, 2014. 